\title{
The Role of Vascular Endothelial Growth Factor in Thrombocytosis in Colorectal Cancer Patients
}

\author{
Dwi Indira Setyorini, ${ }^{1}$ Mediarty, ${ }^{2}$ Irsan Saleh, ${ }^{3}$ Subandrate ${ }^{4 *}$ \\ ${ }^{1}$ Internal Medicine Program, Faculty of Medicine, Universitas Sriwijaya/RSMH Palembang \\ ${ }^{2}$ Department of Internal Medicine, Faculty of Medicine, Universitas Sriwijaya/RSMH Palembang \\ ${ }^{3}$ Department of Pharmacology, Faculty of Medicine, Universitas Sriwijaya \\ ${ }^{4}$ Department of Biochemistry, Faculty of Medicine, Universitas Sriwijaya
}

\author{
Received : January 31 2018 \\ Accepted : March 15 2018
}

*Correspondence :subandrate@unsri.ac.id

\begin{abstract}
Background: Colorectal cancer was included in a group of cancer with various complications. One complication that was often a cause of morbidity and mortality was thrombocytosis. In colorectal cancer, the incidence of thrombocytosis associated with the formation of blood vessels around the tumor or angiogenesis. Factors that played an important role in angiogenesis was vascular endothelial growth factor (VEGF).

Methods: This study was an observational analytic research in colorectal cancer patients to determine the correlation levels of platelets and serum VEGF levels. A total of 33 patients with colorectal cancer at the Palembang Mohammad Hoesin Hospital be research subjects to examine the levels of platelets and levels of VEGF. The level of serum VEGF was performed using ELISA technique from SIGMA®.

Results: The average level of the patient's platelets was $281,090.9 \pm 105,860.8 / \mathrm{mm} 3$. In this study, two patients $(6.06 \%)$ have thrombocytosis. The average serum levels of VEGF research subjects were $221.2 \pm 152.8$ $\mathrm{pg} / \mathrm{mL}$. Correlation test of levels of serum VEGF and platelets levels showed the value of $\mathrm{p}=0.040(\mathrm{p}>0.05)$ and $\mathrm{r}=$ 0468.

Conclusions: Thus, it can be concluded that in this research serum VEGF levels are almost always causes an increase in platelet levels in patients with colorectal cancer.
\end{abstract}

Keywords: Colorectal cancer, platelets, thrombocytosis, VEGF 


\section{BACKGROUND}

Colorectal cancer is cancer that forms from the colon or the rectum, also known as the large intestine. Colorectal cancer is the third most common cancer and cause of cancer deaths in the United States third. American Cancer Society (ACS) 136830 bet that the diagnosis of colorectal cancer and 50310 deaths in 2014. Likewise in Indonesia, colorectal cancer also ranks third incidence of cancer. It has also become another problem in Indonesia because epidemiological studies showed that patients with colorectal cancer at a young age Indonesia is much higher than other developing countries. Health cases in the productive age would become their own health problems in Indonesia., ${ }^{1,2}$

Incidence of thromboembolism is a complication that is often found in malignancy and is a cause of significant morbidity and mortality. Approximately $15 \%$ of all cancer patients experienced thromboembolic disorders that include deep vein thrombosis, pulmonary embolism, thrombosis at the venous access mapun arterial thrombosis. In patients with cancer, thrombosis ranks second as a cause of death. Cancer can affect blood flow through a mechanical effect on the blood vessels surrounding the tumor. In addition it is believed that angiogenesis induced by tumor blood vessels produce complex with abnormal blood flow. Microvascular around the tumor increased permeability to proteins include fibrinogen. One factor that helps the process of angiogenesis is vascular endothelial growth factor (VEGF). ${ }^{3,4}$

VEGF is a glycoprotein proangiogenic that works to increase proliferation, migration, survival in endothelial cells and improve capillary permeability. VEGF can be produced by almost all cells undergoing hypoxia or under stressful conditions, including endothelial cells. And also expressed high in tissues that grow or undergo remodeling as cancer, atherosclerosis, or remodeling in the female reproductive cycle. In addition to increasing the permeability and angiogenesis, VEGF also induces expression of tissue factor (TF) resulting in extravascular fibrin deposition. Instead VEGF production is regulated by the cytoplasmic domain of $\mathrm{TF}^{5-7}$.In cancer, the increased expression of VEGF and the buildup of platelets to form the thrombus has a reciprocal relationship. The process of cancer metastasis creating lesions in the cell. As a result, there will be an increase and withdrawal of platelets to the lesion to form a thrombus. Furthermore, accumulation of thrombus triggers VEGF expresses cancer cells to form new vascular tissue. This situation in turn triggered a hypercoagulation thus worsening the quality of life in cancer patients. ${ }^{8-10}$ Some studies have linked the incident a hypercoagulation with an 
increased serum VEGF. Some hypercoagulation markers such as D-dimer, fibrinogen, and platelet reported to be elevated in cancer patients. Research conducted on breast cancer showed that the expression of VEGF serum and plasma increase with increasing levels of platelets. ${ }^{8-10}$ Looking at the data above and there are no data, especially in Indonesia, on the link between platelet levels with VEGF serum is necessary to do research on the correlation between levels of platelets by VEGF serum in patients with colorectal cancer to administration of adjuvant therapy so that the quality of life of patients with colorectal cancer may increase.

\section{METHODS}

This study is observational analytic research to determine the correlation with serum levels of VEGF and platelet levels in patients with colorectal cancer at the Hospital Muhammad Hoesin Palembang. Patients who become subjects of this study are patients with colorectal cancer who was treated at the Clinic Hematology Disease and patients with colorectal cancer who were treated at the Mohammad Hoesin Palembang Hospital with criteria inclusion is evidenced by the results of the biopsy histopathology in Department Pathology. Criteria for rejection of the research subjects were patients with a history of previous hypercoagulation, diabetes mellitus, patients with a history of stroke, patients who are pregnant and patients who receive anticoagulant therapy.

This study was conducted between August 2016 and February 2017 in the Laboratory of Clinical Pathology, Laboratory of Biochemistry and Biomedical Laboratory Faculty of Medicine, University of Sriwijaya. Blood samples of colorectal cancer patients who meet the inclusion criteria and did not meet the inclusion criteria taken using a 5 cc syringe for examination and inspection VEGF and platelet levels. VEGF examination was performed using ELISA technique in accordance with the procedures from SIGMA®. Data is processed and presentedtabulated and narrative, and then analyzed through Pearson correlation test using SPSS 20. This study was conducted after obtaining a certificate of ethics approval from Unit of Bioethics and Humanities Faculty of Medicine, University of Sriwijaya/RSMH Palembang No. 137/kepkrsmhfkUnsri/2016.

\section{RESULTS}

\section{Characteristics of Research Subjects}

In this study, the research subjects were 35 consisting of 15 men $(42.1 \%)$ and 20 women (57.9\%). The average age of the study subjects was $42.25 \pm 11.8$ years (Table 1 ). 
Table 1.Characteristics of Research Subjects

\begin{tabular}{|c|c|c|c|}
\hline Characteristic & Man & Woman & Average \\
\hline Age & $\begin{array}{l}44,75 \pm 13,7 \text { years } \\
\text { old }\end{array}$ & $40,25 \pm 10$ years old & $\begin{array}{c}42,25 \pm 11,8 \text { years } \\
\text { old }\end{array}$ \\
\hline $\mathrm{N}$ & $15(42,1 \%)$ & $2057,(\%)$ & \\
\hline
\end{tabular}

\section{Platelet levels}

The average platelet levels in this study is $281,090.9 \pm 105,860.8 / \mathrm{mm} 3$. The highest platelet levels was 553,000/mm3 and platelets lowest was $111,000 / \mathrm{mm} 3$.

\section{Serum VEGF levels}

The average serum VEGF levels in this study was $221.2 \pm 152.8 \mathrm{pg} / \mathrm{mL}$. The highest serum VEGF levels are $652 \mathrm{pg} / \mathrm{mL}$ and the lowest serum levels of VEGF was $34 \mathrm{pg} / \mathrm{mL}$.

\section{DISCUSSION}

The average levels of platelets in the subject of research is still in the normal category. The normal value is 200,000-500,000 platelets / $\mathrm{mm} 3$. There are research subjects who had a platelet value under normal or above normal. In this study, the incidence of thrombocytosis of $6.06 \%$. Malignancy or the use of chemotherapy which can cause damage to the vascular endothelium. Malignancy can affect blood flow through a mechanical effect on the blood vessels around the tumor. In addition, angiogenesis induced by tumor blood vessels produce complex with abnormal blood flow. ${ }^{8}{ }^{810}$ Effect of mechanical and process flow angiogenesis increases platelet aggregation processes that increase the tendency to thrombocytosis.Colorectal cancer as one type of malignancy in solid tumors, usually accompanied by changes in hemostatic potential for the formation of thromboembolism. The prevalence of thromboembolic events after 2 years of undetected colorectal cancer ranged from 2.2 to $12 \% .{ }^{9,10}$

There is plenty of other evidence that colorectal cancer and other gastrointestinal cancers induce thrombocytosis and hypercoagulable state. This condition reflects the aggressiveness of cancer cell biology. Induction of platelet production and thrombocytosis caused by 
overexpression of thrombopoietin (TPO) and the release of cytokines, such as IL-1, IL-3, IL-6, IL-11 and other proteins such as granulocyte macrophage colony stimulating factor (GM-CSF), ligand FMS tyrosine kinase 3 (Flt3) and fibroblast growth factor (FGF). In addition, in patients with colorectal cancer increased peripheral platelet networking needs of chronically during the process of formation of micro-emboli. It stimulates the formation of platelets to maintain platelet counts within the normal range. ${ }^{8-10}$

VEGF is glycoprotein proangiogenic that works to increase proliferation, migration, survival in endothelial cells and improve capillary permeability. VEGF can be produced by almost all cells undergoing hypoxia or under stressful conditions, including endothelial cells. And also expressed high in tissues that grow or undergo remodeling as cancer, atherosclerosis, or remodeling in the female reproductive cycle. In addition to increasing the permeability and angiogenesis, VEGF also induces expression of tissue factor (TF) resulting in extravascular fibrin deposition. Instead VEGF production is regulated by the cytoplasmic domain of TF. ${ }^{5-7}$

\section{Correlation between Serum VEGF levels and Platelets Levels}

To determine the correlation Serum VEGF levels of platelets and then performed Pearson correlation test. Pearson correlation test results showed the value of $p=0.04(P<0.05)$ with the strength of the relationship $r=0.468$. These results indicate that platelet levels correlated positively with serum VEGF levels with the strength of the correlation was. This means that any increase in serum VEGF is almost always followed by an increase in platelets. In cancer, the increased expression of VEGF and the buildup of platelets to form the thrombus has a reciprocal relationship. The process of cancer metastasis creating lesions in the cell. As a result, there will be an increase and withdrawal of platelets to the lesion to form a thrombus. Furthermore, accumulation of thrombus triggers VEGF express cancer cells to form new vascular tissue. This situation in turn triggered a hypercoagulable state thus worsening the quality of life in cancer patients. ${ }^{8-10}$ Some studies have linked the incident a hypercoagulablestate with an increased serum VEGF. Some hypercoagulable state markers such as D-dimer, fibrinogen, and platelet reported to be elevated in cancer patients. Research conducted on breast cancer showed that the expression of VEGF serum and plasma increase with increasing levels of platelets. $^{8-10}$ 


\section{CONCLUSION}

In this study it can be concluded that the incidence of thrombocytosis in patients with colorectal cancer in RSMH Palembang is $6.06 \%$. VEGF plays an important role in increasing platelet levels in colorectal cancer patients. Increased levels of VEGF is almost invariably lead to increased levels of platelets or the tendency to thrombocytosis.

\section{BIBLIOGRAPHY}

1. Abdullah M, Sudoyo AW, AR Utomo, Fauzi A, Rani AA. Molecular profiles of colorectal cancer in Indonesia: is there another pathway? Bed Bench GastroenterolHepatol 2012; 5 (2): $71-78$

2. Haggar FA, Boushey RP. Colorectal Cancer Epidemiology: Incidence, Mortality, Survival, and Risk Factors. Clinics In Colon And Rectal Surgery. 2009; 4 (22): 191-197

3. Boccaccio C, Medico E. Cancer and blood coagulation. Cell. Mol. Life Sci. 2006; 63: 10241027

4. Kusmardi PRA. Role of Vascular Endothelial Growth Factor in Proangiogenic on Tumor Angiogenesis. Medicinus. 2011; 3 (6): 15-25

5. Joseph M, Kumar A, Kushwaha R, MLB Bhatt, Chandra A, Kant S, et al. Prospective study on clotting Factors in selected non-hematological malignancies. Biomedical Research, 2013; 24 (4): 445-448

6. Bates DO. Vascular endothelial growth factors and vascular permeability. Cardiovascular Research. 2010; 87: 262-271

7. Olsson AK, Dimberg A, Kreuger J, Claesson-Welsh L. VEGF receptor signaling - in control of vascular function. Molecular Cell Biology. 2006 7: 359-371

8. Selheim F, Holmsen H, Vassbotn FS. Identification of functional human VEGF receptors on platelets. Elsevier BV. 2002; 512: 107-110

9. Kirwan CC, Byrne GJ, Kumar S, McDowell G. Platelet release of Vascular Endothelial Growth Factor (VEGF) in Patients undergoing chemotherapy for breast cancer. Journal of Angiogenesis Research. 2009; 1 (7): 1-9 
10. Schwarzbach CJ, Schaefer A, Ebert A, Held V, Bolognese M; Kablau M, et al. The Importance of Cancer-Associated Hypercoagulation as a Possible Stroke etiology. Stroke. 2012; 43: 3029-3034 